\title{
Performance Modeling of Proposed GUISET Middleware for Mobile Healthcare Services in E-Marketplaces
}

\author{
Alaba Olu Akingbesote, Mathew Olusegun Adigun, Sibisuso Xulu, and Edgar Jembere \\ Department of Computer Science, University of Zululand, Private Bag X1001, KwaDlangezwa 3886, South Africa \\ Correspondence should be addressed to Alaba Olu Akingbesote; oluwamodimu2012@gmail.com
}

Received 27 June 2014; Accepted 8 October 2014; Published 18 November 2014

Academic Editor: Yuh-Rau Wang

Copyright (C) 2014 Alaba Olu Akingbesote et al. This is an open access article distributed under the Creative Commons Attribution License, which permits unrestricted use, distribution, and reproduction in any medium, provided the original work is properly cited.

\begin{abstract}
GUISET is a proposed middleware engine currently under study in South Africa. The goal is to provide utility services for small, medium, and macroenterprises in the context of mobile e-services. Three things are important to make this engine effective and efficient: the implementation, performance, and the pricing strategy. The literature has delved richly into implementation issue of similar projects. Both the performance and the pricing strategy issues have not been fully discussed especially in the context of mobile healthcare services. Some literature has addressed the performance issue using the exogenous nonpriority and the preemptive model. However, with providers offering different services using that approach may prove to be difficult to implement. This work extends existing and widely adopted theories to non-preemptive model by using the queuing theory and the simulation model in the context of mobile healthcare services. Our evaluation is based on non-preemptive priority and nonpriority discipline. Our results reveal that the unconditional average waiting time remains the same with reduction in waiting time over the nonpreemptive priority model in four out of the five classes observed. This is envisaged to be beneficial in mobile healthcare services where events are prioritized and urgent attention is needed to be given to urgent events.
\end{abstract}

\section{Introduction}

The e-marketplaces are local community of service providers and requestors (service consumers) organized in vertical markets and gathering around portals [1]. These emarketplaces have allowed consumers to shop for bread of services from anywhere in the world based on pay-as-you-go model [2].

One major improvement in these e-markets is the issue of mobile services whereby the data processing and storage are moved from the mobile device to powerful and centralized computing platforms and then accessed over the wireless connection based on a thin native client or web browser on the mobile devices [3]. This improvement brought the idea of Grid Based Utility Infrastructure Software Engineering Technology (GUISET) project.

The basic goal of GUISET is to provide utility infrastructure services especially in the context of mobile healthcare services to small, medium, and microenterprise. These mobile e-services include e-health (healthcare service), ecommerce, and e-learning. The idea of this project is centered on the fact that mobile devices (e.g., smartphone and tablet PC) are increasingly becoming an essential part of human life. They are the most effective and convenient communication tools not bounded by time and place. Mobile users accumulate rich experience of various services from mobile applications (e.g., iPhone apps and Google apps), which run on their devices and/or on remote servers via wireless networks. With rapid growth in mobile technology both in low- and middle-income countries, particularly in Africa and in the less affluent and rural communities, mobile device access is far greater than access to computers in developing countries. For instance, in South Africa about 50 million people have mobile phone access and everyday experience confirms a near-universal mobile ownership, with everyone from street vendors to top-level executives carrying one [4].

The report of World Health Organization (WHO) global survey on e-health indicates that the dominant form of 
$\mathrm{m}$-health today is characterized by small-scale pilot projects that address single issues in information sharing and access. This report also noted the existence of a number of applications gaining attraction for mobile phone-based health issues [5].

In addition, mobiles services have been attracting the attention of entrepreneurs as a profitable business option that reduces the development and running cost of mobile applications of mobile users as a new technology to achieve rich experience of a variety of mobile services at low cost and of researchers as a promising solution for green IT [6]. Three things are important to make this engine effective and efficient: the GUISETs implementation, performance, and the pricing strategy. While the implementation and pricing strategy process are on course, see for example, [7-9], that of performance is yet to be fully addressed. For example, in the International Data Cooperation (IDC) report, the performance challenge of Cloud which is a similar project rose in 2008 from $63.1 \%$ to $82.9 \%$ in 2009 as reported in [10]. This is an increase of $19.8 \%$ as against the Security challenge of about $12.9 \%$.

One important aspect of performance is the waiting time to respond to consumers by e-Cloud providers for products or requests. This waiting time, which is a key source of competitive advantage, is so important that traditional marketplaces like MCDonalds in 1990s offered consumers their meal free of charge if the order was not served within 2 minutes [11].

The focus of this research is to investigate the proposed GUISET middleware performance based on requests waiting time of mobile events with different service offerings in the context of e-health; that is, we study and evaluate the service discipline to be adopted in the context of mobile e-health where providers of service have different service offerings for mobile aware requests.

Most existing literature that delved into performance impact, for example, the Cloud e-marketplaces, focuses on the exogenous nonpriority model with emphasis on First Come First Served discipline [12-14] or Shortest Job Next [15]. As the server farms increase with consumers demanding different service disciplines, some scholars like [16] use the preemptive service discipline in the context of Cloud emarketplaces but literature reveals that, in practice, preemption and migration of virtual machines are costly [17]. Second, preemption leads to increase in response time of consumers' requests especially when the requests are deadline constrained [18]. This work extends existing and widely adopted theories to non-preemptive model in the context of the proposed GUISETs middleware with emphasis on mobile e-health.

Our assumption in this paper is that each medical point of call is called server and we then follow that of $[14,19]$ that the system consists of a number of server machines that are allocated to patients' request based on the service discipline. Furthermore, each mobile event request is assigned to only one server, and each server can only run at most one task at a time.

The remainder of this paper is organized as follows. Section 2 discusses the related work. Section 3 introduces our analytical model description with the numerical and simulation setup. In Section 4, we have our results and discussion. We have the conclusion in Section 5.

\section{Literature Review}

Since this is a proposed project and yet to be fully implemented, we study existing literature of a similar project which has given us the opportunity to extend the body of knowledge and we focus on Cloud issue on performance.

In [20], the authors study the effect of queuing in relation to the time spent by patients to access clinical services multiuser model. The authors analyze the data and the results of the analysis show that average queue length, waiting time of patients, and overutilization of doctors at the clinic could be reduced at an optimal server level.

In [12], the authors address three things; these are the levels of QoS that can be guaranteed given service resources, the number of service resources that are required to ensure that customer services can be guaranteed in terms of the percentile, and the number of customers to be supported to ensure that customer services can be guaranteed in terms of the percentile of response time. The work of [19] uses the $\mathrm{M} / \mathrm{G} / \mathrm{c}$ to evaluate a Cloud server firm with the assumption that the numbers of server machines are not restricted. The result of the authors demonstrates the manner in which request response time and number of tasks in the system may be assessed with sufficient accuracy.

The work of [14] uses the $\mathrm{M}^{[x]} / \mathrm{G} / \mathrm{m} / \mathrm{m}+\mathrm{r}$ to describe a new approximate analytical model for performance evaluation of Cloud data centers with batch task arrivals. The results show that important performance indicators such as mean request response time, waiting time in the queue, queue length, blocking probability, probability of immediate service, and probability distribution of the number of tasks in the system can be obtained in a wide range of input parameters. This work is based on the so-called on-demand service.

In [16], the authors propose a preemptive policy in Cloud market. The idea is that when an urgent request arrives, it preempts the current request in service and such preempted request is then migrated to another virtual machine if it cannot meet the deadline for completion. In [18], the authors remove the scheduling bottleneck from one-dimensional to multidimensional resources. This is done with the use of Multidimensional Resource Integrated Scheduling (MRIS) which is an inquisitive algorithm to obtain the approximate optimal solution.

The work of [15] proposes $\mathrm{M} / \mathrm{M} / \mathrm{m}$ queuing model to develop a synthetic optimization method to optimize the performance of services in an on-demand service. The simulation result shows that the proposed method can allow less waiting time and queue length and more customers to gain the service using synthetic optimization function when the number of servers increases. In [21], the authors model the Cloud using $\mathrm{M} / \mathrm{M} / \mathrm{c} / \mathrm{c}$ model with different priority classes with the main goal of studying the rejection probability for different priority classes. But [22] extends Kleinrock's analysis to derive the stationary waiting distribution for each class 


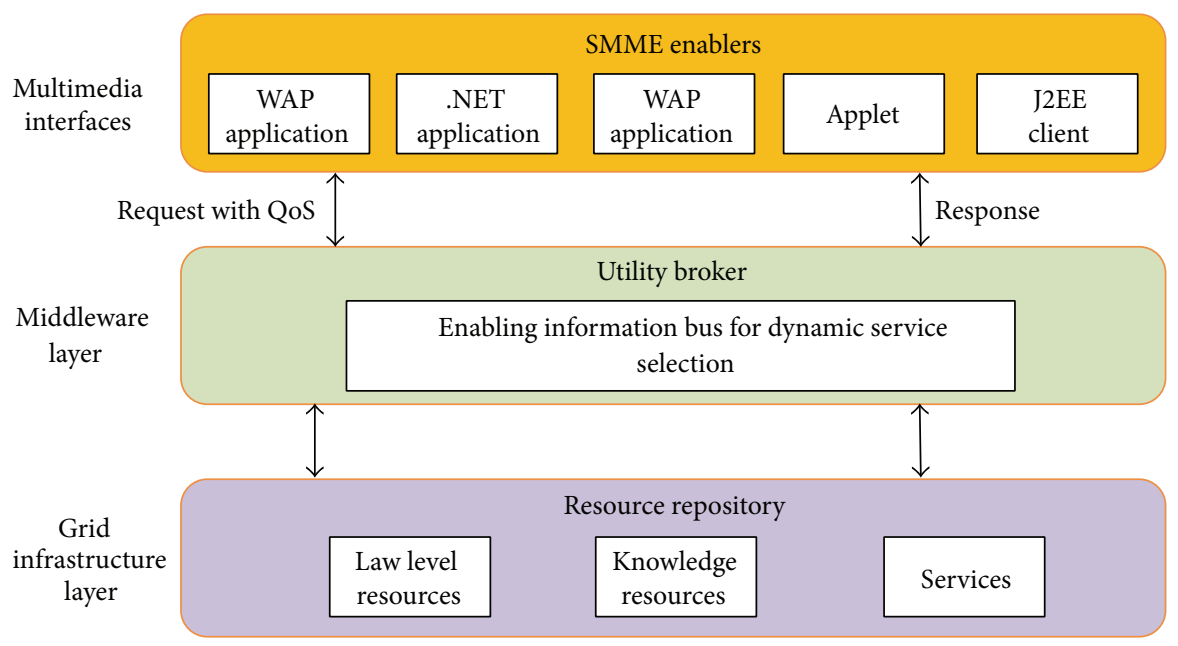

FIGURE 1: GUISET architecture.

in a single server accumulating priority queue with Poisson arrival and general distribution service time.

All these authors have made one or more contributions to the existing knowledge. These works have given us the opportunity to make our contribution based on the few observations we noticed. For instance, the works of $[12,14$, $15,20,22]$ were based on FCFS or SJN which could not work in e-health mobile service where the e-mobile events are prioritized. Also, the generalized approach used by [12] could not reflect the real Cloud market of today. The work of [16] produces a good result but [17] reveals that preemption and migration of virtual machines are costly.

Our work is closely related to $[23,24]$ where these authors model the Cloud as series of queues. What differentiate our work are the following:

(i) each of our service stations is modelled as $\mathrm{M} / \mathrm{M} / \mathrm{c} / \mathrm{Pr}$ as against the $\mathrm{M} / \mathrm{M} / 1$ proposed by these authors;

(ii) no dedicated server is given or allocated to any class, thereby reducing consumers' waiting time;

(iii) our research is in the context of mobile services.

\section{The General GUISETs Architecture and the Proposed Middleware Model}

We present two architectures; the first is the general architecture of the proposed GUISETs in Figure 1 and the second is our proposed performance GUISET middleware prototype model in the context of e-health in Figure 2. The second is calved out from the first as part of the services.

The GUISETs architecture has three major layers: the multimodal layer, the middleware, and the infrastructural layer. The multimodal layer consists of SMME enabling applications which we refer to as the client side. This includes the WAP, Net, and other applications as shown in Figure 1. The clients send mobile aware requests to the GUISET middleware and the middleware does the service discovery, selection, and composition. Apart from these it checks for authentication and authorization and resolves the issue of pricing strategy; it ensures the provisioning of good quality of service through better service discipline. The third layer is the infrastructure layer that contains the resource repository. These are low level, knowledge, and other services like ehealth service.

Our research is in this middleware where we focus on the service discipline to be adopted in the context of mobile e-health where providers of service have different service offerings for mobile aware requests.

Our proposed prototype GUISET model is shown in Figure 2. The health-aware mobile devices are connected to the mobile networks via a base station, for example, through base transceiver station or access point that establishes and controls the connections and functional interfaces between the networks and mobile devices. We gather our health information from [25-27]. The mobile user information (e.g., ID and location, BS, BP) is recorded and the risk column field is classified into five levels that determine the mobile event class as shown in Table 1. For example, mobile event that has blood sugar greater than $200 \mathrm{mg} / \mathrm{d}$ or blood pressure of $180 / 110$ and above is classified as severe or very high risk with class 1 and that between 140-200 and 160-169/100-109 is classified as moderate or high risk with class 2 . These e-health mobile events are classified under five categories in this research as shown in Table 1. These are transmitted to the central processor which is the dispatcher-in server that provides authentication, authorization, order of request (service discipline), and accounting services based on the home agent and subscribers' data stored in databases. After that, the requests are delivered to the right destination where the e-health mobile network services will be provided and results are then dispatched back through the dispatcherout. One assumption is that the mobile gargets and the access points are already in place; secondly, the issues of authentication, authorization, and accounting services are out of scope of this research. Our research is to prioritize these classes so that urgent requests get access to solution faster than the less urgent ones. The priority is such that the order is in the form of class 1 mobile aware event having higher 
TABLE 1: The e-health mobile aware records.

\begin{tabular}{|c|c|c|c|c|c|c|}
\hline \multirow{2}{*}{ ID } & \multirow{2}{*}{ Location } & \multirow{2}{*}{ Blood sugar (mg/dL) } & \multicolumn{2}{|c|}{ Blood pressure ( $\mathrm{mmHg}$ ) } & \multirow{2}{*}{ Risk } & \multirow{2}{*}{ Mobile event class } \\
\hline & & & Systolic & Diastolic & & \\
\hline XXXX1 & Ngoma & $>200$ & $\geq 180$ & $\geq 110$ & Very high (severe) & $\mathrm{C} 1$ \\
\hline $\mathrm{XXXX} 2$ & Ongoye & $>140$ but $<200$ & $160-179$ & $100-109$ & High (moderate) & $\mathrm{C} 2$ \\
\hline XXXX3 & Empangeni & $\geq 126 \leq 139$ & $140-159$ & $90-99$ & Mild & $\mathrm{C} 3$ \\
\hline $\mathrm{XXXX} 4$ & Esikhawini & 100 but $<126$ & $120-139$ & $80-89$ & Warning & $\mathrm{C} 4$ \\
\hline XXXX5 & Richards Bay & 110 & 120 & 80 & No (normal) & C5 \\
\hline
\end{tabular}

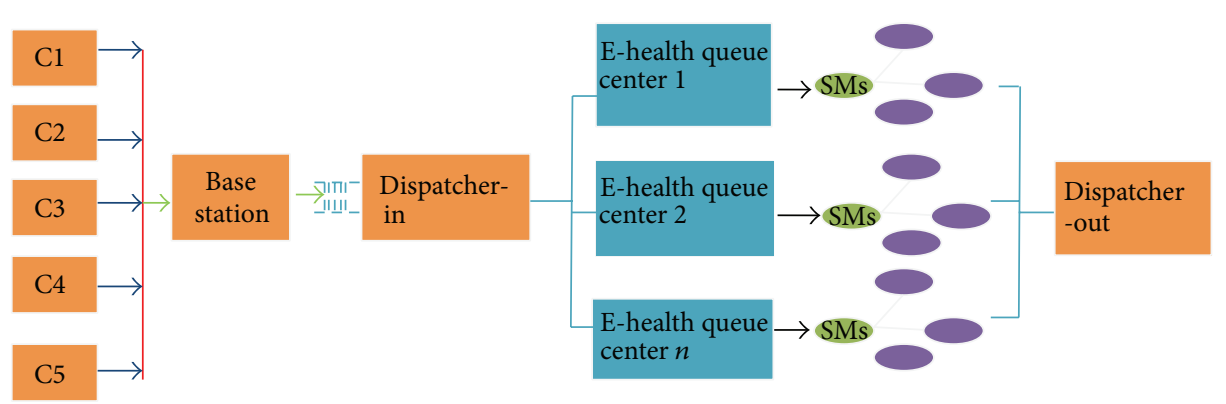

FIGURE 2: Proposed GUISET mobile healthcare model.

priority than classes $2,3,4$, and 5 . When an incoming request meets lower one on the queue, it takes over that request but when lower request is already underprocessed, that request has to be completed. When requests of the same priority are on the queue then the order is based on First Come First Served (FCFS). All requests are sent to the dispatcher-in server where they are then distributed to the e-health center stations for processing. The processed request then moves through the dispatcher-out as the outlet.

Unlike most related works $[16,22,24]$ where the nonpreemptive policy is implemented at the first point of entry alone, we model our non-preemptive model at every point of queue, that is, the dispatcher-in queue as $\mathrm{M} / \mathrm{M} / 1 / \mathrm{Pr}$, the ehealth centers as $\mathrm{M} / \mathrm{M} / \mathrm{c} / \mathrm{Pr}$, and the dispatcher-out queue as $\mathrm{M} / \mathrm{M} / 1 / \mathrm{Pr}$. This is because at every point of queue there is likely tendency that higher priority will arrive when lower one is on the queue.

This proposed model assumes that the arrival and the service process are exponentially distributed and due to large number of consumers entering the market for request we assume an infinite population. To get our performance measure, we follow the law of conservation of flow and the steps stated in [28].

3.1. Mathematical Modelling of Dispatcher-In Queue. Let mobile event with service of the $k$ th priority (the smaller the number, the higher the priority) arrive before the dispatcherin according to Poisson distribution with parameter $\lambda_{k}(k=$ $1,2, \ldots, r)$ and these consumers wait on First Come First Served basis within their respective priorities. Let the service distribution for the $k$ th priority be exponential with mean $1 / \mu_{k}$. As earlier said, whatever the priority of a unit in service is, it has to complete its service before another item is admitted.
We define

$$
\begin{gathered}
\rho_{k}=\frac{\lambda_{k}}{\mu_{k}}, \quad(1 \leq k \leq r), \\
\sigma_{k}=\sum_{i=1}^{k} \rho_{k}, \quad\left(\sigma_{0} \equiv 0, \sigma_{r} \equiv \rho\right) .
\end{gathered}
$$

The system stationary for $\sigma_{r}=\rho<1$. Let an event of priority $i$ arrives at time $t_{0}$ and enters service at time $t_{1}$. Its line wait is thus $T_{q}=t_{1}-t_{0}$. At $t_{0}$ let us say we have $n_{1}$ of priority one in line ahead of this new arrival, $n_{2}$ of priority two, $n_{3}$ of priority three, and so on. Let $S_{0}$ be the total time required to finish the job already in service and $S_{k}$ the total time required to serve $n_{k}$. During the new mobile request waiting time $T_{q}$ (say) $n_{k}^{\prime}$ mobile request of priority $k<1$ will arrive and go to service ahead of this current arrival. If $S_{k}^{\prime}$ is the total service time of all $n_{k}^{\prime}$, then it can be seen that

$$
T_{q}=\sum_{k=1}^{i-1} S_{k}^{\prime}+\sum_{k=1}^{i} S_{k}+S_{0}
$$

taking the expected values from both sides of the foregoing, then

$$
W_{k}^{\prime}=E\left[T_{q}\right]=\sum_{k=1}^{i-1} E\left[S_{k}^{\prime}\right]+\sum_{k=1}^{i} E\left[S_{k}\right]+E\left[S_{0}\right] .
$$

Since $\sigma_{i-1}<\sigma_{i}$ for all $i$, then $\rho<1$ implies that $\sigma_{i-1}<1$ for all $i$.

To find $E\left[S_{0}\right]$, we observe that the combined service distribution is the mixed exponential, which is formed from the law of total probability as

$$
B(t)=\sum_{k=1}^{i} \frac{\lambda_{k}}{\lambda}\left(1-e^{-\mu_{k} t}\right),
$$


where

$$
\lambda=\sum_{k=1}^{r} \lambda_{k}
$$

The random variable remaining time of service $S_{0}$ has the value 0 when the system is idle; hence

$$
E\left[S_{0}\right]=\operatorname{Pr}\{\text { system is busy }\} E\left[S_{0} \mid \text { busy system }\right] .
$$

But the probability that the system is busy is

$$
\lambda=\sum_{k=1}^{r} \frac{\lambda_{k}}{\lambda} \frac{1}{\mu_{k}}=\rho
$$

where $\rho$ is the server utilization and

$$
\begin{aligned}
& E\left[S_{0} \mid \text { busy system }\right] \\
& =\sum_{k=1}^{r} \lambda_{k} E\left[S_{0} \mid \text { system busy wit } k \text { type cutomer }\right] \\
& \quad \cdot \operatorname{Pr}\{\text { mobile request has priority } k\} \\
& =\sum_{k=1}^{r} \frac{1}{\mu_{k}} \frac{\rho_{k}}{\rho} ;
\end{aligned}
$$

therefore,

$$
E\left[S_{0}\right]=\rho \sum_{k=1}^{r} \frac{1}{\mu_{k}} \frac{\rho_{k}}{\rho}=\sum_{k=1}^{r} \frac{\rho_{k}}{\rho} .
$$

Since $n_{k}$ and the service times of individual mobile requests $S_{k}^{(n)}$ are independent,

$$
E\left[S_{k}\right]=E\left[n_{k} S_{k}^{(n)}\right]=E\left[n_{k}\right] E\left[S_{k}^{(n)}\right]=\frac{E\left[n_{k}\right]}{\mu_{k}} .
$$

Utilizing Little's formula then gives

$$
E\left[S_{k}\right]=\frac{\lambda_{k} W_{q}^{(k)}}{\mu_{k}}=\rho_{k} W_{q}^{(k)} ;
$$

similarly,

$$
E\left[S_{k}^{\prime}\right]=\frac{E\left[n_{k}^{\prime}\right]}{\mu_{k}}
$$

and then utilizing the uniform properties of the Poisson we have

$$
E\left[S_{k}^{\prime}\right]=\frac{E\left[n_{k}\right]}{\mu_{k}} \frac{\lambda_{k} W_{q}^{(i)}}{\mu_{k}} ;
$$

therefore $W_{q}^{(i)}$ which is the waiting time for mobile request of $i$ priority is

$$
\begin{aligned}
& W_{q}^{(i)}=W_{q}^{(i)} \sum_{k=1}^{i-1} \rho_{k}+\sum_{k=1}^{i} \rho_{k} W_{q}^{(k)}+E\left[S_{0}\right], \\
& W_{q}^{(i)}=\frac{\sum_{k=1}^{i} \rho_{k} W_{q}^{(k)}+E\left[S_{0}\right]}{1-\sigma_{i-1}}, \\
& W_{q}^{(i)}=\frac{E\left[S_{0}\right]}{\left(1-\sigma_{i-1}\right)\left(1-\sigma_{i}\right)} .
\end{aligned}
$$

Using (9) finally gives

$$
W_{q}^{(i)}=\frac{\sum_{k=1}^{r} \rho_{k} / \mu_{k}}{\left(1-\sigma_{i-1}\right)\left(1-\sigma_{i}\right)} .
$$

The above equation holds as long as $\sigma_{i}=\sum_{k=1}^{r} \rho_{k}<1$.

Therefore, from Little's formula,

$$
L_{q}^{(i)}=\sum_{k=1}^{r} \lambda_{i} W_{k}^{(i)}=\frac{\lambda_{i} \sum_{k=1}^{r} \rho_{k} / \mu_{k}}{\left(1-\sigma_{i-1}\right)\left(1-\sigma_{i}\right)} .
$$

The total expected system size in each of the single channels is

$$
L_{q}=\sum_{i=1}^{r} L_{q}^{i}=\frac{\lambda_{i} \sum_{k=1}^{r} \rho_{k} / \mu_{k}}{\left(1-\sigma_{i-1}\right)\left(1-\sigma_{i}\right)}
$$

3.2. Mathematical Modelling of Mobile E-Health Center. Unlike the $M / M / 1 / \operatorname{Pr}$, the $M / M / c / P r$ is governed by identical exponential distributions for each priority at each of the $c$ channels within a station. As earlier said, our service rate is equal throughout the experiment. We define

$$
\begin{gathered}
\rho_{k}=\frac{\lambda_{k}}{c \mu_{k}}, \quad(1 \leq k \leq r), \\
\sigma_{k}=\sum_{i=1}^{k} \rho_{k}, \quad\left(\sigma_{0} \equiv \rho=\frac{\lambda}{c \mu}\right),
\end{gathered}
$$

where the system is stationary for $\rho<1$, and

$$
W_{q}^{(i)}=\sum_{k=1}^{i-1} E\left[S_{k}^{\prime}\right]+\sum_{k=1}^{i} E\left[S_{0}\right]
$$

where $S_{k}$ is the time required to serve $n_{k}$ mobile requests of the $k$ th priority in the line ahead of the consumer. $S_{k}^{\prime}$ is the service time of the $n_{k}^{\prime}$ consumers of priority $k$ which arrive during $W_{q}^{(i)} ; S_{0}$ is the amount of time remaining until the next server becomes available.

Therefore,

$E\left[S_{0}\right]$

$=\operatorname{Pr}($ all servers are busy within a service station $)$

- $E\left[S_{0} \mid\right.$ all server are busy within a service station $]$

$$
\begin{aligned}
& =\left(\sum_{n-c}^{\infty} P_{n}\right) \frac{1}{c \mu} \\
& =P_{0}\left(\sum_{n-c}^{\infty} \frac{c \rho^{n}}{c^{n-c} c !}\right) \frac{1}{c \mu}=\frac{P_{0}(c \rho)^{c}}{c !(1-p)} \\
& =\frac{(c \rho)^{c}}{c !(1-\rho)(c \mu)}\left[\sum_{n=0}^{c-1} \frac{(c \rho)^{n}}{n !}+\frac{(c \rho)^{c}}{c !(1-\rho)}\right]^{-1}
\end{aligned}
$$


but

$$
\begin{gathered}
E\left[S_{0}\right]=\rho \sum_{k=1}^{r} \frac{1}{u_{k}} \frac{\rho_{k}}{\rho}=\sum_{k=1}^{r} \frac{\rho_{k}}{u_{k}}, \\
W_{q}^{(i)}=\frac{E\left[S_{0}\right]}{\left(1-\sigma_{i-1}\right)\left(1-\sigma_{i}\right)}, \\
W_{q}^{(i)}=\frac{\sum_{k=1}^{r}\left(\rho_{k} / u_{k}\right)}{\left(1-\sigma_{i-1}\right)\left(1-\sigma_{i}\right)} .
\end{gathered}
$$

Therefore

$$
\begin{aligned}
W_{q}^{(i)} & =\frac{\left[c !(1-\rho)(c \mu) \sum_{n=0}^{c-1}(c \rho)^{(n-c) / n !}+c \mu\right]^{-1}}{\left(1-\sigma_{i-1}\right)\left(1-\sigma_{i}\right)}, \\
T W_{q s t}^{(i)} & =\frac{E\left[S_{0}\right]}{\left(1-\sigma_{i-1}\right)\left(1-\sigma_{i}\right)} \\
& =\frac{\left[c !(1-\rho)(c \mu) \sum_{n=0}^{c-1}(c \rho)^{(n-c) / n !}+c \mu\right]^{-1}}{\left(1-\sigma_{i-1}\right)\left(1-\sigma_{i}\right)} .
\end{aligned}
$$

The expected time taken in a service station is

$$
W_{q s t}=\sum_{i=1}^{r} \frac{\lambda_{i}}{\lambda} W_{q s t}^{(i)} .
$$

The overall average time taken in $j$ service stations is

$$
W(\text { ave })_{q s t}=\frac{\sum_{n=1}^{j}\left[\sum_{i=1}^{r}\left(\lambda_{i} / \lambda\right) W_{q}^{(i)}\right]}{j} .
$$

3.3. Mathematical Modelling of Dispatcher-Out. Our dispatcher-out is similar to the dispatcher-in; therefore the waiting time is derived using (1) to (15) and (18). We obtain

$$
W_{q}^{T \text { dispout }}=\sum_{i=1}^{r} W_{q}^{i} .
$$

The total waiting time by, say, class $i$ priority is

$$
W_{q}^{i \text { tot }}=W_{q \mathrm{din}}^{i}+W_{q \mathrm{st}}^{i}+W_{q \mathrm{dout}}^{i}
$$

while the total waiting time experienced in the queue by the whole classes is

$$
w_{q}=\sum_{n=1}^{m} W_{q}^{i \operatorname{tot}^{n}}
$$

3.4. Numerical Validation and Simulation. First, we validate our mathematical solution with the simulation to ascertain the degree of correction. This is done by considering five classes of consumers' arrival. These are represented as five arrival processes $\lambda_{1}, \lambda_{2}, \lambda_{3}, \lambda_{4}$, and $\lambda_{5}$, where $\lambda_{1}=\lambda_{2}=$ $\lambda_{3}=\lambda_{4}=\lambda_{5}$ and $\lambda=\lambda_{1}+\lambda_{2}+\lambda_{3}+\lambda_{4}+\lambda_{5}$.

We use the Wolfram Mathematica 9.0 as the mathematical tool for our validation results and arena 14.5 as the simulator.
TABLE 2: Simulation and analytical.

\begin{tabular}{lcc}
\hline$\rho$ & Sim & Ana \\
\hline 0.1 & 0.000681 & 0.000681 \\
0.2 & 0.001502 & 0.001502 \\
0.3 & 0.002612 & 0.002612 \\
0.4 & 0.004006 & 0.004006 \\
0.5 & 0.00592 & 0.00592 \\
0.6 & 0.008751 & 0.0089 \\
0.7 & 0.013447 & 0.013447 \\
0.8 & 0.021933 & 0.023019 \\
0.9 & 0.047009 & 0.0496 \\
\hline
\end{tabular}

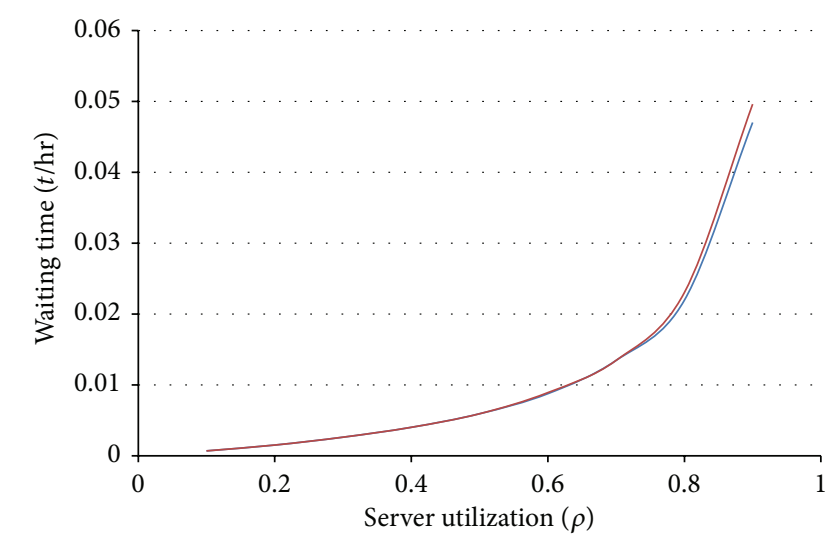

Simulation

FIgURE 3: Simulation and Analytical.

We set $\lambda=100,200,300, \ldots, 970$, respectively, and $c=$ 2 in each of the service stations. We then simulate this experiment using Arena discrete event simulator version 14.5 with the same values to ascertain the degree of variability. This simulation was run with replication length of 1000 in 24 hours per day with base time in hours and it was replicated 5 times. The service rate was set to 0.001 for the dispatcher-in and 0.0005 for each of the servers in the web queue stations and the dispatcher-out.

In addition, we set up a similar experiment with the same configurations but under a nonpriority discipline using FCFS discipline. The illustration of this model is shown by numerical examples and the impact on the five performances of the priority classes is analyzed under the Results and Discussion section.

\section{Results and Discussion}

The analytical results and that of our simulation are compared and the results are shown in Table 2 and Figure 3, respectively. The degree of variation is hardly noticed until $\rho \rightarrow 1$ but the coefficient of variation is very small and less than unity; that is why, in Figure 3, the analytical colour overshadowed the simulation colour at the initial stage. Table 3 provides the results 


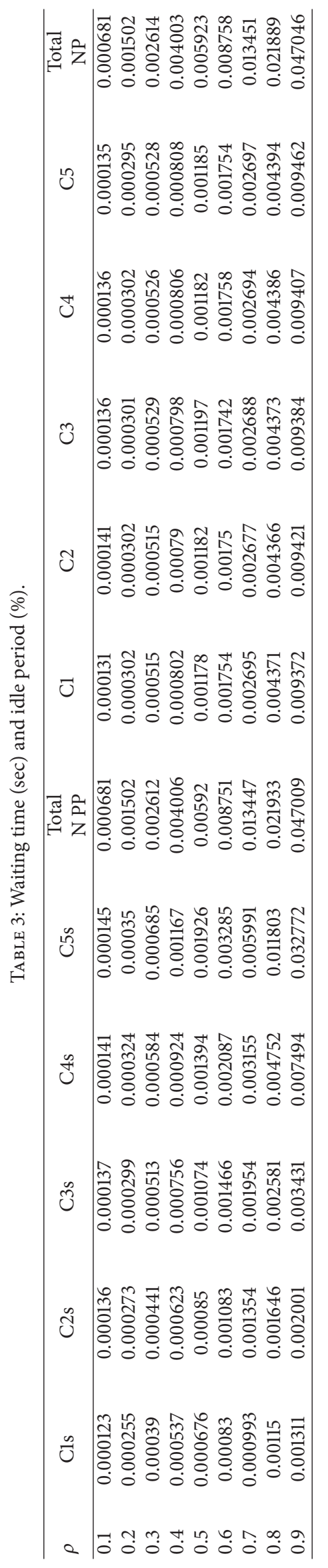




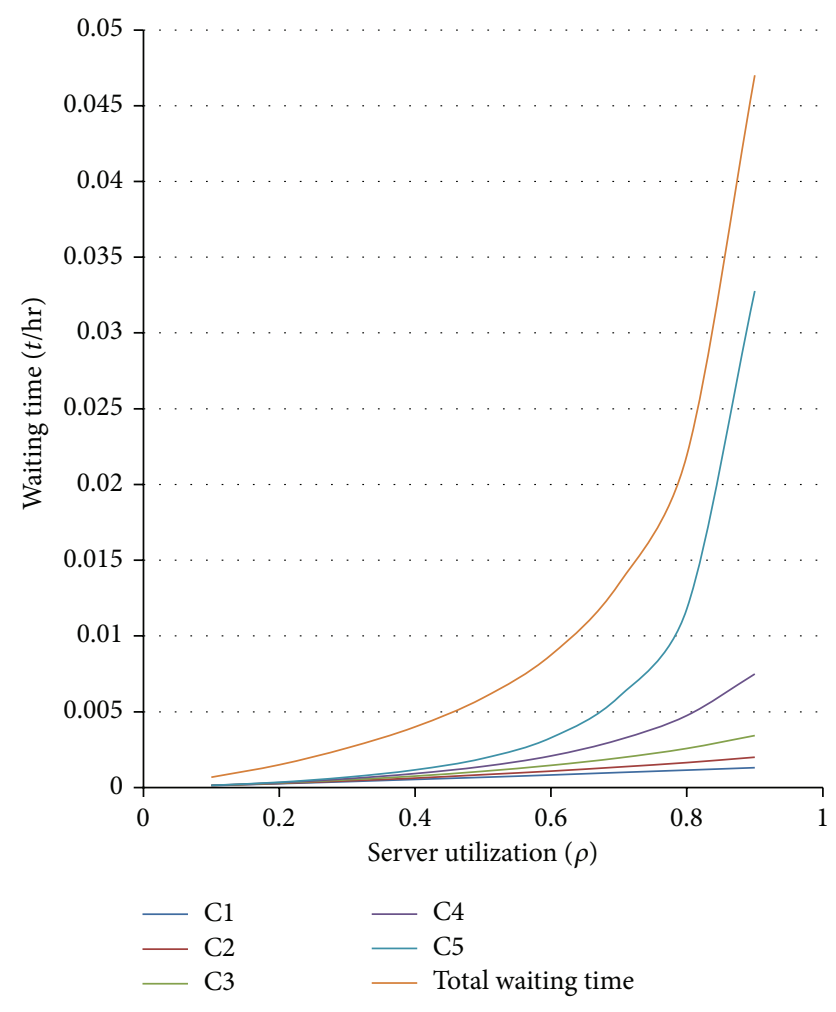

FIgURE 4: Performance of the five classes waiting with the total waiting time.

of our waiting time distributions of our simulation under the non-preemptive priority and the exogenous nonpriority where we use the FCFS policy. We represent the simulation results of non-preemptive as $\mathrm{C} 1 \mathrm{~s}-\mathrm{C} 5 \mathrm{~s}$ and the nonpriority (FCFS) ones as C1-C5. Our first observation reveals that the total waiting time simulation results of both are the same. This implies that the total waiting time on the queue is independent of the service discipline. Second, the waiting time distributions of the classes in the non-preemptive differ while those of nonpriority have equal distributions.

Figure 4 is the performance result of our simulation that we obtained from Table 3 of our non-preemptive priority. At the initial state when the server utilization is below 0.5 the performance difference of these five classes is not fully noticed. As the server utilization increases, that is, the number of mobile events increases in the proposed GUISETs e-marketplace, the performance differentiation becomes well noticed with class 1 priority having the minimum waiting time followed by class two while class five has the highest waiting time. All these four classes have better performance at the expense of the fifth class.

In Figure 5, we compare this model with that of nonpriority model using the First Come First Served policy. Both of them have an unnoticeable performance differentiation below 0.4 but as the $\rho \rightarrow 1$ where $\rho<1$, four classes out of the five classes observed have better waiting time performances over the conventional exogenous nonpriority model. Though the fifth class has higher waiting time, this class can be dedicated to requests that do not pose much risk

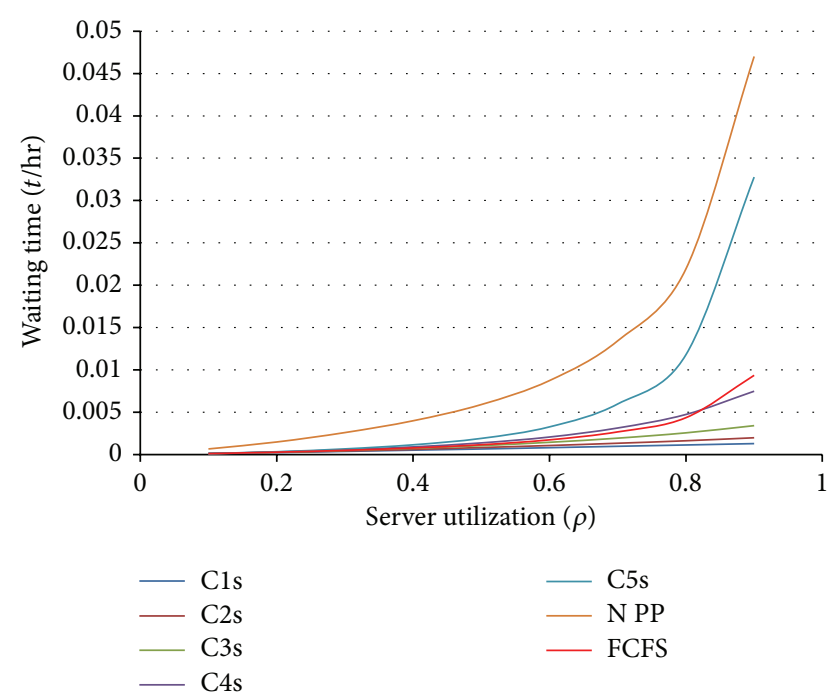

FIGURE 5: Performance of non-preemptive priority and nonpriority.

like checking information whose waiting time does not pose risk, for example, the issue of blood pressure request with $120 / 80$, which is normal in this experiment. This implies that urgent e-health mobile requests have better response time than noncritical requests. We envisage this proposed system to be good in the mobile e-health sector where patients with urgent request equipment need urgent attention. Also, it will be good to the e-health provider if the costs paid per class are prioritized since they have the same overall average waiting time.

\section{Conclusion}

One of the objectives of the proposed GUISET engine is the provisioning of mobile e-services to clients. In this research, we model a typical GUISET mobile healthcare middleware and evaluate the performance impact of mobile healthcare device requests' waiting time using the nonpreemptive priority and the nonpriority service discipline. In our evaluation, we recorded the same average time for both disciplines but the waiting time distributions of each class in the non-preemptive priority differ with the lowest class having the minimum waiting time. Four out of the five classes observed perform better in the non-preemptive discipline than nonpriority discipline. This prototype system will be a good service in the mobile healthcare environment where urgent requests are given higher priority than lower requests. For example, request of severe risk should have higher priority than that of moderate or mild risk.

More can still be done to have better consumers waiting time performance for the whole class. For example, one can consider the case where the lowest priority consumers represent a scheduled task and the highest appear at random.

\section{Conflict of Interests}

The authors declare that there is no conflict of interests regarding the publication of this paper. 


\section{Acknowledgments}

This work is based on the research supported in part by the National Research Foundation of South Africa-Grant UID: TP11062500001 (2012-2014). The authors also acknowledge funds received from industry partners: Telkom SA Ltd, Huawei Technologies SA (Pty) Ltd, and Dynatech Information Systems, South Africa, in support of this research.

\section{References}

[1] P. M. Papazoglou, Web Services: Principle and Technology, Pearson Education, Edinburgh Gate, UK, 2008.

[2] A. O. Akingbesote, M. O. Adigun, J. Oladosu, E. Jembere, and I. Kaseeram, "The Trade-off between consumer's satisfaction and resource service level by e-market providers in e-market places," in Proceedings of the International Conference Electrical Engineering and Computer Sciences (EECS '13), Hong Kong, 2013.

[3] H. T. Dinh, C. Lee, D. Niyato, and P. Wang, "A survey of mobile cloud computing: architecture, applications, and approaches," Wireless Communications and Mobile Computing, vol. 13, no. 18, pp. 1587-1611, 2013.

[4] June 2014, http://www.who.int/bulletin/volumes/90/5/12020512/en/.

[5] World Health Organization, "mHealth: new horizons for health through mobile technologies," in Global Observatory for eHealth Services, vol. 3, WHO, 2011, http://www.who.int/\%20goe\%20/ publications/eHealth_series_vol3/en/.

[6] M. Ali, "Green cloud on the horizon," in Proceedings of the 1st International Conference on Cloud Computing (CloudCom '09), pp. 451-459, Manila, Philippines, 2009.

[7] T. Shezi, E. Jembere, and M. Adigun, "Towards developing failure tolerant communication framework for GUISET services," in Proceedings of the Southern Africa Telecommunication Networks and Applications Conference (SATNACT '11), 2011.

[8] E. K. Olatunji, M. O. Adigun, E. Jembere, J. Oladosu, and P. Tarwire, "A privacy-as-a-service model for securing data in GUISET environment," in Southern Africa Telecommunication Networks and. Applications Conference (SATNAC '13), September 2013.

[9] M. E. Buthelezi, M. O. Adigun, O. O. Ekabua, and J. S. Iyilade, "Accounting, pricing and charging service models for a GUISET grid-based service provisioning environment," in Proceedings of the International Conference on e-Learning, eBusiness, Enterprise Information Systems, and e-Government (EEE '08), pp. 350-355, July 2008.

[10] S. O. Kuyoro, F. Ibikunle, and O. Awodele, "Cloud computing security issues and challenges," International Journal of Computer Networks, vol. 3, no. 5, 2011.

[11] C. C. Kim, L. Smith, H. Thorne, and R. W. Hilton, "Managing costs and time for customer value," in Management Accounting: Information for Managing and Creating Value, chapter 15, pp. 726-728, McGraw-Hill, Maidenhead, UK, 2008.

[12] K. Xiong and H. Perros, "Service performance and analysis in cloud computing," in Proceedings of the World Conference on Services-I, pp. 693-700, Los Angeles, Calif, USA, July 2009.

[13] H. Khazaei, J. Mišić, and V. B. Mišić, "Performance analysis of cloud centers under burst arrivals and total rejection policy," in Proceedings of the Global Telecommunications Conference (GLOBECOM '11), pp. 1-6, IEEE, Houston, Tex, USA, December 2011.
[14] H. Khazaei, M. Jelena, and B. M. Vojislav, "Performance evaluation of cloud data centers with batch task arrivals," in Communication Infrastructures for Cloud Computing, pp. 199223, 2013.

[15] L. Guo, T. Yan, S. Zhao, and C. Jiang, "Dynamic performance optimization for cloud computing using $\mathrm{M} / \mathrm{M} / \mathrm{m}$ queueing system," Journal of Applied Mathematics, vol. 2014, Article ID 756592, 8 pages, 2014.

[16] R. Santhosh and T. Ravichandran, "Pre-emptive scheduling of on-line real time services with task migration for cloud computing," in Proceedings of the International Conference on Pattern Recognition, Informatics and Mobile Engineering (PRIME '13), pp. 271-276, February 2013.

[17] S. T. Maguluri, R. Srikant, and L. Ying, "Stochastic models of load balancing and scheduling in cloud computing clusters," in Proceedings of the IEEE Conference on Computer Communications (INFOCOM '12), pp. 702-710, March 2012.

[18] S. Nagendram, J. V. Lakshmi, D. V. N. Rao, and C. N. Jyothihi, "Efficient resource scheduling in data centers using MRIS," Indian Journal of Computer Science and Engineering, vol. 2, no. 5, 2011.

[19] P. Ehsan and P. Massoud, "Minimizing data center cooling and server power costs", in Proceedings of the 14th ACM/IEEE International Symposium on Low Power Electronics and Design, pp. 145-150, New York, NY, USA, 2009.

[20] M. M. Kembe, E. S. Onah, and S. Iorkegh, "A study of waiting and service costs of a multi-server queuing model in a specialist hospital," International Journal of Scientific \& Technology Research, vol. 1, no. 8, pp. 19-23, 2012.

[21] W. Ellens, M. Živković, J. Akkerboom, R. Litjens, and H. Van Den Berg, "Performance of cloud computing centers with multiple priority classes," in Proceedings of the IEEE 5th International Conference on Cloud Computing (CLOUD '12), pp. 245-252, Honolulu, Hawaii, USA, June 2012.

[22] D. A. Stanford, P. Taylor, and I. Ziedins, "Waiting time distributions in the accumulating priority queue," Queueing Systems, vol. 77, no. 3, pp. 297-330, 2014.

[23] X. Nan, Y. He, and L. Guan, "Optimal resource allocation for multimedia cloud based on queuing model," in Proceedings of the 13th International Workshop on Multimedia Signal Processing (MMSP '11), pp. 1-6, IEEE, Hangzhou, China, October 2011.

[24] F. Kamoun, "Performance analysis of a non-preemptive priority queuing system subjected to a correlated Markovian interruption process," Computers \& Operations Research, vol. 35, no. 12, pp. 3969-3988, 2008.

[25] http://www.nhlbi.nih.gov/hbp/detect/categ.htm.

[26] http://www.idph.state.ia.us/hpcdp/common/pdf/diabetes/classfinal.pdf.

[27] Primary health care, Standard Treatment Guidelines and Essentail Medicines List, Health Department, Western Cape, South Africa, 2008, http://www.kznhealth.gov.za/edlphc2008.pdf.

[28] G. Donald and M. H. Carl, Fundamentals of Queueing Theory, John Wiley \& Sons, 2nd edition, 1985. 


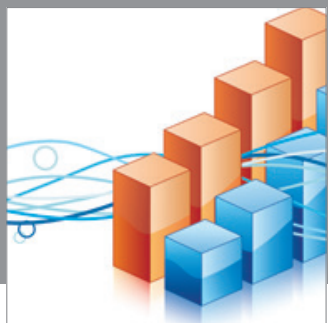

Advances in

Operations Research

mansans

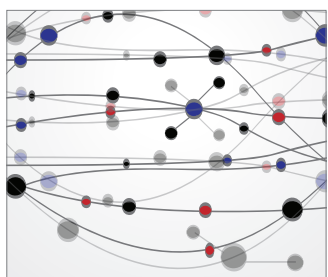

The Scientific World Journal
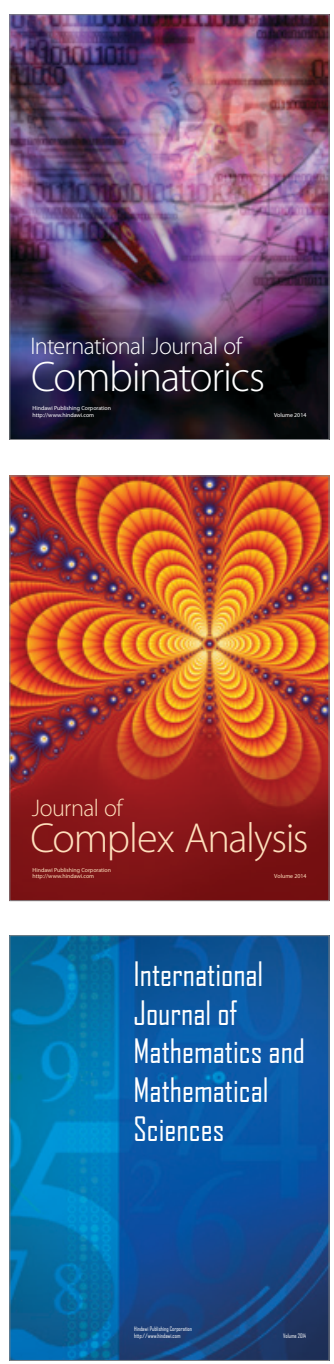
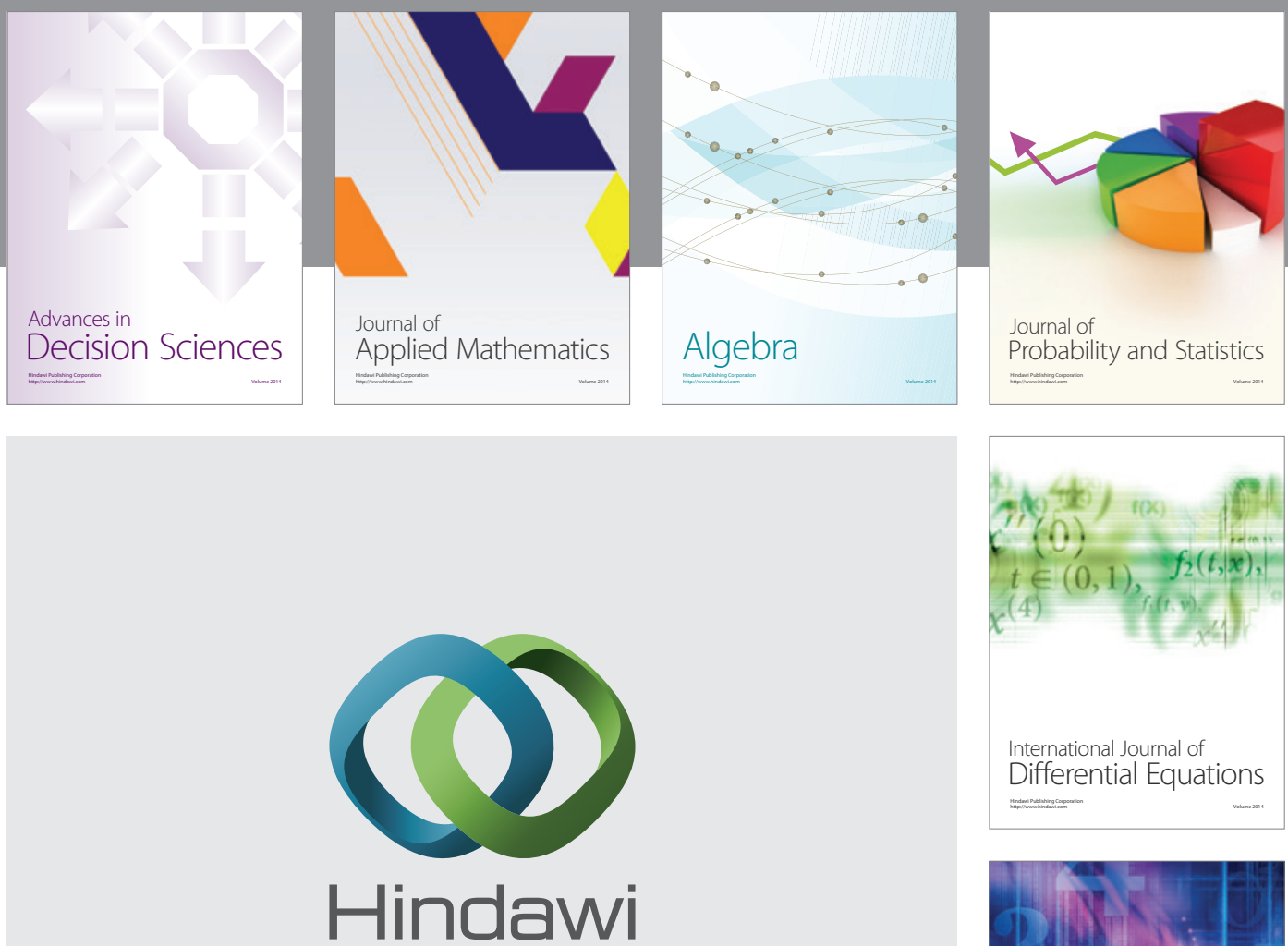

Submit your manuscripts at http://www.hindawi.com
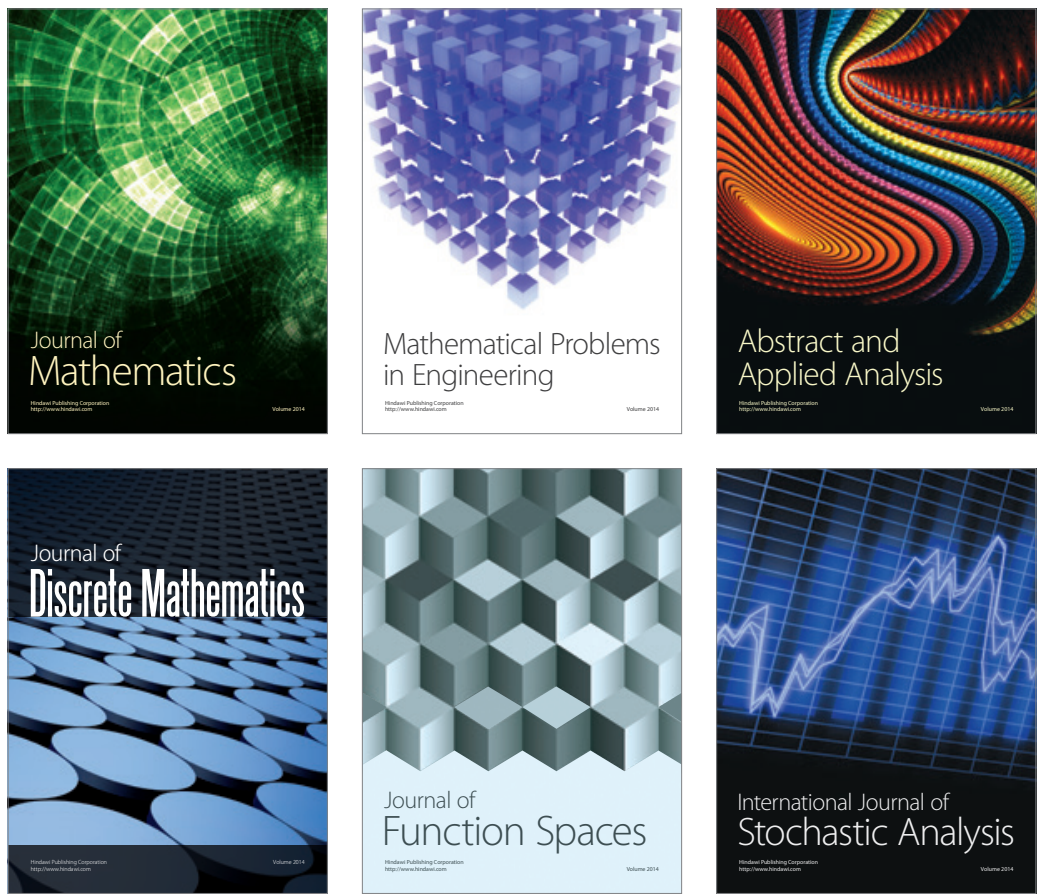

Journal of

Function Spaces

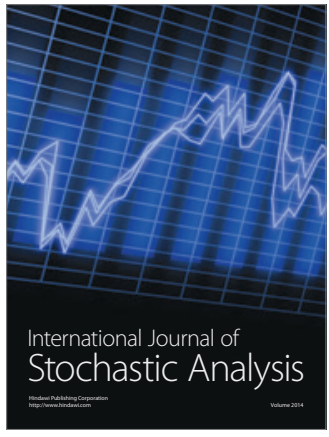

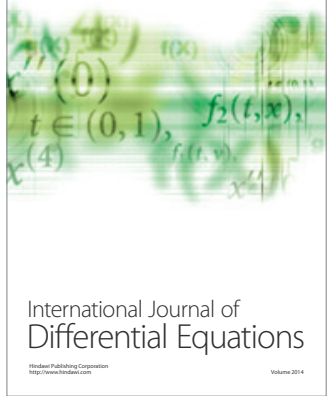
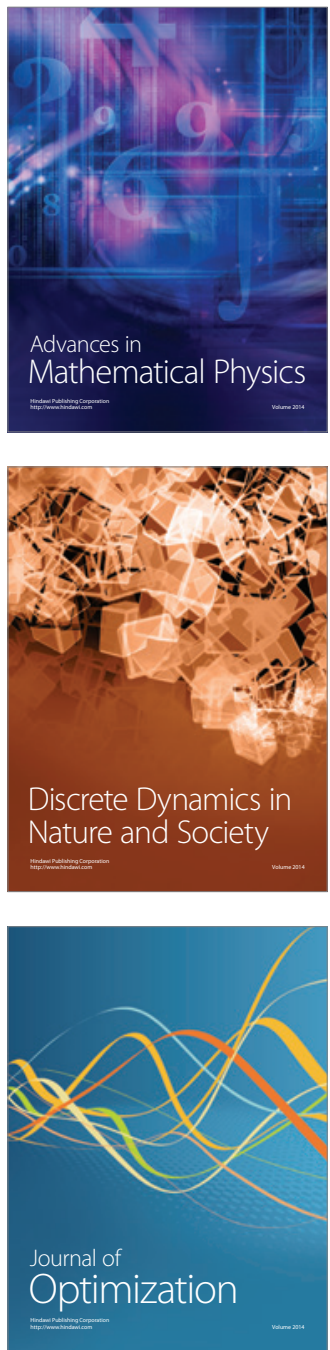